\title{
Risco reprodutivo e renda familiar: análise do perfil de gestantes
}

\author{
Reproductive risk and family income: \\ analysis of the profile of pregnant women
}

Rozania Bicego Xavier ${ }^{1}$ Claudia Bonan Jannotti ${ }^{2}$ Katia Silveira da Silva ${ }^{2}$ Aline de Carvalho Martins ${ }^{1}$
${ }^{1}$ Departamento de Obstetrícia, Instituto Nacional de Saúde da Mulher, da Criança e do Adolescente Fernandes Figueira, Fiocruz. Av. Rui Barbosa 716, Flamengo. 22250-020 Rio de Janeiro RJ.r.bicego@ig.com.br ${ }^{2}$ Programa de PósGraduação em Saúde da Mulher e da Criança, Instituto Nacional de Saúde da Mulher, da Criança e do Adolescente Fernandes Figueira, Fiocruz.

\begin{abstract}
Faced with the challenge of tackling maternal and perinatal morbidity and mortality, the discussion of reproductive risk and its association with health vulnerability situations is relevant to public health. The research carried out at Instituto Fernandes Figueira/Fiocruz sought to describe the reproductive risk profile of women admitted to prenatal care between 2006 and 2008 and investigating its relation to family income and health vulnerability situations. This is a crosssectional study which used the prenatal care database as its source. The economic profile of the group under scrutiny is a low income group. An association between low income and fetal malformations, pregnancy before 15 years of age, and HIV was detected. A higher family income was associated with the mother's age over 35 years, abnormal nuchal translucency, gynecopathies, and habitual abortion. The findings may reflect vulnerability situations of low income women, such as unfavorable health conditions and limited access to and use of health services. It is necessary to acquire in-depth knowledge of the reproductive risk profile in Brazil and its distribution according to socio-demographic characteristics, seeking to enhance health care practices provided.
\end{abstract}

Key words High-risk pregnancy, Risk factors, Family income, Health vulnerability
Resumo Em face do desafio de enfrentar a morbimortalidade materna e perinatal, a discussão sobre risco reprodutivo e sua associação com situações de vulnerabilidade em saúde é relevante à saúde publica. A pesquisa realizada teve como objetivos descrever o perfil de risco reprodutivo de 3440 mulheres matriculadas em um serviço de pré-natal de alto risco, entre 2006 e 2008, e investigar sua relação com renda familiar e situações de vulnerabilidade em saúde. Trata-se de estudo transversal que teve como fonte o banco de dados do pré-natal. O perfil econômico do grupo estudado é de baixa renda. Encontrou-se associação entre baixa renda familiar e malformações fetais, gravidez antes dos 15 anos e HIV. Renda familiar mais elevada foi associada à idade materna acima de 35 anos, translucência nucal alterada, ginecopatias e abortamento habitual. Os achados podem refletir situações de vulnerabilidade de mulheres de baixa renda, como condições de saúde desfavoráveis e menor acesso e utilização de serviços de saúde. É necessário obter um maior conhecimento do perfil do risco reprodutivo no Brasil e sua distribuição segundo características sociodemográficas, visando ao aperfeiçoamento das práticas assistenciais.

Palavras-chave Gestação de alto risco, Fatores de risco, Renda familiar, Vulnerabilidade de saúde 


\section{Introdução}

No ano 2000, a Organização das Nações Unidas promoveu conferência internacional para analisar os principais problemas mundiais e traçar estratégias para a promoção do desenvolvimento social e a superação das iniquidades entre países e regiões do mundo. Conhecida como Cúpula do Milênio, a conferência estabeleceu oito grandes Objetivos de Desenvolvimento do Milênio, entre os quais figuram a redução da mortalidade na infância e a melhoria da saúde das gestantes.

A redução da mortalidade materna em 75\% no período entre 1990 e 2015 é uma das metas pactuadas pelos 191 países participantes da Cúpula do Milênio. Para cumprir esses compromissos e garantir direitos previstos na Constituição Federal, o Ministério da Saúde tem proposto uma série de diretrizes, normas e protocolos para incrementar a qualidade da atenção ao pré-natal, ao parto e ao puerpério ${ }^{1-5}$ e melhorar os indicadores de saúde materna e neonatal.

No Brasil, entre 1990 e 2007, houve um decréscimo de $46 \%$ nas taxas de mortalidade materna, ou seja, de 140 para 75 óbitos por $100 \mathrm{mil}$ nascidos vivos ${ }^{6}$. Entretanto, essa redução foi observada principalmente na década de 1990; nos anos 2000, as taxas de mortalidade materna mantiveram-se em um patamar ainda elevado, de cerca de 74 óbitos por 100 mil nascidos vivos. As taxas de mortalidade infantil, por sua vez, apresentaram importante declínio nas últimas décadas, porém a mortalidade neonatal, intimamente relacionada com a assistência ao pré-natal e ao parto, se mantém estabilizada em níveis altos ${ }^{7}$.

Os indicadores de saúde materna são considerados muito sensíveis às desigualdades sociais, refletindo condições diferenciadas de vida e de acesso a recursos sociais (saúde, educação, renda, trabalho, segurança, participação) entre diversos grupos da população, e as formas como classe social, gênero e raça/etnia se entrelaçam e operam como determinantes sociais da saúde ${ }^{8-}$ ${ }^{11}$. No Brasil, a maioria das mortes e morbidades maternas severas é devida a causas consideradas evitáveis, como hipertensão, hemorragia, infecção ou complicações de abortamento ${ }^{12}$. Isso significa que o acesso a uma assistência à saúde oportuna, humanizada e de boa qualidade evitaria que muitas mulheres perdessem suas vidas por motivos reprodutivos ${ }^{11,12}$.

O marco conceitual da vulnerabilidade em saúde, proposto por Ayres et al. ${ }^{13}$ é profícuo para a análise do complexo fenômeno da saúde e da morbimortalidade materna. Os autores realçam a relação intrínseca entre individuo e coletivo, em contextos específicos, no processo de saúde-adoecimento, e consideram que as situações de vulnerabilidade envolvem três dimensões interdependentes: individual, social e programática. A vulnerabilidade individual diz respeito a informações, redes de relacionamento e outros recursos sociais que os indivíduos dispõem e lhe conferem maior ou menor habilidade de agir mediante problemas de saúde; a vulnerabilidade social remete às macroestruturas sociais e suas formas de redistribuição de recursos cognitivos e materiais e de oportunidades de participação e reconhecimento social; a vulnerabilidade programática se refere à forma como as instituições respondem e atendem ou não às demandas e necessidades de saúde dos diferentes sujeitos e grupos sociais. Na interdependência desses três planos, se configuram os determinantes sociais de saúde, e o modelo conceitual de vulnerabilidade em saúde é útil não somente para analisar os processos de saúde e adoecimento, como também nos convida pensar criticamente e renovar as práticas de saúde.

Essa perspectiva tem sido incorporada nas normas e diretrizes das políticas de assistência à saúde de gestantes de alto risco. O Manual Técnico de Gestação de Alto Risco ${ }^{5}$ destaca a importância de uma abordagem integral às mulheres, considerando-se questões de gênero, raça/etnia e classe social, e, preconiza o manejo adequado de "situações de vulnerabilidade relacionadas ao processo saúde-doença, sejam elas individuais, sociais e até mesmo programáticas". O Ministério da Saúde propõe uma classificação operacional dos fatores de risco reprodutivo, distribuindo-os em quatro categorias: características individuais e condições sociodemográficas desfavoráveis; história reprodutiva anterior; condições clínicas preexistentes e intercorrências clínicas; e doença obstétrica na gravidez atual. Várias condições elencadas no primeiro grupo indicam situações de vulnerabilidade individual e/ou social - como nível de escolaridade, conflitos familiares ou conjugais, condições de trabalho desfavoráveis, exposição a ambientes insalubres e abuso de drogas - que, muitas vezes, manifestam desigualdades e discriminações, seja por classe social, gênero, cor de pele, idade ou outras. A interdependência entre vulnerabilidade programática e risco reprodutivo também é discutida no manual que ressalta que "é importante que as mulheres em idade reprodutiva, especialmente aquelas em situações de vulnerabilidade, tenham acesso aos serviços de saúde e oportunidade de estar bem informadas e na melhor condição física pos- 
sível antes de engravidar. Como exemplo podemos citar uma mulher diabética, que deve estar bem controlada antes de engravidar".

Essa classificação é uma ferramenta importante para identificação e manejo de riscos durante o pré-natal e também para uma abordagem de riscos reprodutivos em mulheres não gestantes, em idade fértil. Entretanto, o perfil do risco reprodutivo na população brasileira ou em subgrupos populacionais e sua correlação com situações de vulnerabilidade ainda é pouco estudado no Brasil. Em busca realizada na base de dados Literatura Latino-Americana e do Caribe em Ciências da Saúde (Lilacs), utilizando o descritor "gestação de alto risco" foram encontrados 329 artigos publicados entre março de $2002 \mathrm{e}$ março de 2012. A maioria deles volta-se para a discussão de questões clínico-obstétricas envolvidas na gestação de alto risco e realizadas durante o período do pré-natal, parto ou puerpério. Um percentual pequeno $(4,3 \% ; 14$ artigos) de estudos relacionou características socioeconômicas (escolaridade, renda, trabalho, gênero, raça/etnia, violência, tabagismo, álcool) com a gestação de alto risco, e um número ainda menor ( 9 artigos; $2,7 \%$ ) teve como foco a discussão da relação do contexto de vulnerabilidade social com este desfecho, de forma semelhante ao proposto nesta pesquisa.

O presente artigo apresenta um estudo realizado no ambulatório de pré-natal do Instituto Nacional da Saúde da Mulher, da Criança e do Adolescente Fernandes Figueira da Fundação Oswaldo Cruz (IFF/Fiocruz) que atende gestantes de alto risco, cujos objetivos foram descrever o perfil de risco reprodutivo das gestantes matriculadas no serviço entre 2006 e 2008, investigar sua relação com renda familiar e discutir situações de vulnerabilidade em saúde.

\section{Materiais e métodos}

Realizou-se um estudo de corte transversal, tendo como fonte o banco de dados do ambulatório de pré-natal do IFF/Fiocruz. Esse instituto é uma das unidades de referência para gravidez de alto risco fetal no Estado do Rio de Janeiro. Em 2006, frente à necessidade de aprimorar as formas de registro de informações das gestantes que se matriculam no pré-natal, a equipe do setor criou um banco de dados, composto de um único formulário, que é preenchido no momento da admissão da usuária. Ele permite a coleta de dados de identificação pessoal das usuárias, infor- mações sociodemográficas e aspectos do histórico reprodutivo, registro de patologias clínicas, infecciosas ou obstétricas pregressas, informações sobre a gestação atual e os motivos de encaminhamento para pré-natal de alto risco, classificação do risco gestacional no momento da admissão. Foram incluídas no estudo todas as gestantes matriculadas no ambulatório de pré-natal entre janeiro de 2006 a dezembro 2008, correspondendo a 3440 mulheres.

Para este estudo, foram consideradas todas as variáveis sociodemográficas disponíveis (idade, local de residência, escolaridade, renda familiar), informações sobre número de gestações, partos e abortos espontâneos e inseguros, idade gestacional na admissão no IFF e a classificação de risco (alto ou baixo). Com exceção desta última, todas as demais variáveis foram reagrupadas de modo que permitissem a comparabilidade com outros estudos.

As informações sobre os motivos para encaminhamento ao pré-natal de alto risco foram recategorizadas segundo a classificação dos fatores de risco reprodutivo proposta no Manual Técnico Gestação de Alto Risco ${ }^{5}$ e organizadas em torno das quatro categorias já citadas: a) características individuais e condições sociodemográficas desfavoráveis, b) história reprodutiva anterior, b) condições clínicas preexistentes e intercorrências clínicas, d) doença obstétrica na gravidez atual.

Foi feita uma descrição da distribuição proporcional das características sociodemográficas e reprodutivas das gestantes. Em seguida, realizou-se a descrição do perfil dos fatores considerados de risco reprodutivo, identificando os mais frequentes e aquelas patologias com importante impacto na morbimortalidade materna, como antecedente de DHEG/eclâmpsia, sífilis, rubéola, toxoplasmose, diabetes prévio e diabetes gestacional, pré-eclâmpsia e eclâmpsia.

Diante das informações disponíveis no banco de dados, a avaliação do contexto de vulnerabilidade social foi realizada principalmente a partir da análise do nível de renda da população. Em relação à vulnerabilidade individual e programática, optou-se por observar o local de residência da população e o perfil de risco reprodutivo em relação à idade, à história reprodutiva anterior e às condições clínicas preexistentes, pois estas características permitem realizar uma reflexão sobre o acesso, a oferta e a demanda de serviços de saúde.

Para investigar a associação das variáveis que caracterizam o risco reprodutivo com a renda 
familiar, realizou-se análise bivariada através do teste de Qui-quadrado $\left(\mathrm{X}^{2}\right)$, cujo nível de significância definido foi de 5\%. Foram incluídos nesta análise somente os fatores de risco cuja ocorrência na amostra estudada foi maior do que 30 casos. Os grupos referentes a outras patologias infecciosas e não infecciosas e outras doenças obstétricas da gravidez atual reúnem um universo muito heterogêneo de doenças e, por isso, não foram incluídos na investigação da associação. Utilizou-se o programa Epi Info version 3.5.1 para tabulação dos dados e análises estatísticas.

Neste estudo foram observadas as normas da Comissão Nacional de Ética em Pesquisa (CONEP) do Conselho Nacional de Saúde. O trabalho de campo teve início somente após a aprovação do projeto pelo Comitê de Ética em Pesquisa do Instituto Fernandes Figueira.

\section{Resultados}

Nos período estudado, inscreveram-se no ambulatório de pré-natal do IFF/Fiocruz 3440 gestantes. A idade das mulheres variou de 12 a 48 anos, com média de 27 anos. A maior concentração de gestantes estava na faixa etária entre 20 e 35 anos $(59,6 \%)$, e as demais se dividiam entre adolescentes e mulheres em idade materna avançada. No momento da admissão no ambulatório de pré-natal, $83,3 \%$ das gestações foram classificadas como de "alto risco" e 16,5\% de "baixo risco", estando estas últimas distribuídas por todas as faixas etárias, exceto aquelas extremas (menos de 15 anos e mais de 35 anos). Dois terços dessa população residiam no município do Rio de Janeiro e as demais em outros municípios ou estados (Tabela 1 ).

A análise dos dados de escolaridade foi limitada, pois não havia essa informação para $57 \%$ das mulheres. Considerando as demais gestantes (1462 mulheres), cerca $60 \%$ possuíam no máximo o primeiro grau completo. A maioria $(62,6 \%)$ declarou renda menor ou igual a três salários mínimos, incluindo 7,6\% cujas famílias viviam com menos de um salário mínimo. Apenas $27 \%$ tinham renda familiar igual ou superior a quatro salários mínimos e para $10 \%$ dos casos, não havia essa informação (Tabela 1).

No momento da matrícula no IFF, 33,5\% das mulheres estavam em sua primeira gestação e $22 \%$ eram grandes multigestas (história de mais de três gravidezes), com um caso de 15 gestações. Em relação à paridade, $43 \%$ dessas mulheres não tinham história de parto, $46 \%$ referiram um ou
Tabela 1. Distribuição proporcional das características sociodemográficas e reprodutivas de gestantes atendidas no ambulatório de pré-natal de alto risco do IFF/Fiocruz. 2006-2008.

\begin{tabular}{|c|c|c|}
\hline & \multicolumn{2}{|c|}{$\begin{array}{l}\text { Número } \\
\text { total de } \\
\text { mulheres } \\
=3440\end{array}$} \\
\hline & $\mathbf{N}$ & $\%$ \\
\hline \multicolumn{3}{|l|}{ Idade } \\
\hline 12-19 anos & 749 & 21,8 \\
\hline 20-35 anos & 2051 & 59,6 \\
\hline 36-48 anos & 640 & 18,6 \\
\hline \multicolumn{3}{|l|}{ Local de residência } \\
\hline Outro município/estado & 1300 & 37,8 \\
\hline Rio de Janeiro & 2135 & 62,0 \\
\hline \multicolumn{3}{|l|}{ Escolaridade } \\
\hline Até $1^{\circ}$ grau completo & 611 & 17,8 \\
\hline $2^{\circ}$ grau incompleto ou completo & 732 & 21,2 \\
\hline Superior incompleto ou incompleto & 119 & 3,5 \\
\hline Não informado & 1978 & 57,5 \\
\hline \multicolumn{3}{|l|}{ Renda familiar } \\
\hline Menos de 1 salário mínimo & 260 & 7,6 \\
\hline 1-3 salários mínimos & 1890 & 55,0 \\
\hline 4 ou mais salários mínimos & 935 & 27,1 \\
\hline Não informado & 355 & 10,3 \\
\hline \multicolumn{3}{|l|}{ Número de gestações } \\
\hline 1 & 1151 & 33,5 \\
\hline $2-3$ & 1509 & 43,9 \\
\hline 4 ou mais & 778 & 22,6 \\
\hline \multicolumn{3}{|l|}{ Paridade } \\
\hline 0 & 1465 & 42,5 \\
\hline 1 ou 2 & 1580 & 46,0 \\
\hline 3 ou mais & 394 & 11,4 \\
\hline \multicolumn{3}{|l|}{ Número de filhos vivos } \\
\hline 0 & 1662 & 48,3 \\
\hline $1-2$ & 1483 & 43,1 \\
\hline 3 ou mais & 295 & 8,6 \\
\hline \multicolumn{3}{|l|}{ Histórico de aborto } \\
\hline Não & 2366 & 68,8 \\
\hline 1 & 708 & 20,5 \\
\hline 2 ou mais & 366 & 10,7 \\
\hline \multicolumn{3}{|l|}{ Aborto espontâneo } \\
\hline Sim & 818 & 23,8 \\
\hline Não & 2622 & 76,2 \\
\hline \multicolumn{3}{|l|}{ Aborto inseguro } \\
\hline Sim & 319 & 9,3 \\
\hline Não & 3121 & 90,7 \\
\hline \multicolumn{3}{|l|}{ Início do pré-natal no IFF } \\
\hline Menos de 14 semanas & 947 & 27,5 \\
\hline 14-19 semanas & 788 & 22,9 \\
\hline 20-24 semanas & 566 & 16,4 \\
\hline $25-30$ semanas & 620 & 18,0 \\
\hline Mais de 30 semanas & 514 & 14,9 \\
\hline \multicolumn{3}{|l|}{ Alto risco gestacional } \\
\hline Sim & 2866 & 83,3 \\
\hline Não & 568 & 16,5 \\
\hline Não informado & 6 & 0,2 \\
\hline
\end{tabular}


dois partos e $27 \%$ tinham no mínimo 3 partos, sendo que 27 mulheres tinham histórias de 6 a 10 partos (Tabela 1).

O abortamento foi um evento relativamente frequente nessa população. Cerca de 31,2\% das mulheres informaram algum episódio, sendo que $10,7 \%$ tiveram essa experiência duas ou mais vezes. Abortamentos espontâneos foram relatados por $23,8 \%$ das mulheres e abortos em condições inseguras por $9,3 \%$.

A distribuição de mulheres pela idade gestacional em que iniciaram o pré-natal no IFF revelou que $27,5 \%$ foram matriculadas na unidade com menos de 14 semanas, $22.9 \%$, entre 14 e 19 semanas, $34,4 \%$ entre 20 e 30 semanas (período em que se realiza a ultrassonografia morfológica e que muitas malformações são detectadas), e $14.9 \%$ tardiamente, com mais de 30 semanas de gestação.

Na Tabela 2, podemos observar o perfil de risco reprodutivo da população estudada. A ida- de materna avançada (gestantes com mais de 35 anos) foi o motivo da matrícula no pré-natal de risco em $19 \%$ dos casos, e gravidez no primeiro período da adolescência (de 10 a 14 anos) representou $2,6 \%$ do total.

Cerca de 28\% das mulheres apresentavam história de agravos em gestações anteriores que sinalizavam risco na gravidez atual. Antecedentes de DHEG ou eclâmpsia apareceram em 1,7\% dos casos, de prematuridade e baixo peso em 13,8\%, de malformações fetais em $11,1 \%$ e de neomorto e natimorto em $12,2 \%$, e abortamento habitual em 2,9\%. Um terço das mulheres desse grupo tinha histórico de dois ou mais desses agravos.

As condições clínicas preexistentes e intercorrências clínicas mais frequentes foram as ginecopatias, a hipertensão arterial crônica, o HIV e a toxoplasmose, registradas em cerca de 3\% dos casos. Outras condições como sífilis, rubéola e diabetes prévio, que têm sido alvos de preocupações específicas no campo da saúde pública, apa-

Tabela 2. Perfil do risco reprodutivo de gestantes atendidas no ambulatório de pré-natal de alto risco do IFF/ Fiocruz. 2006-2008.

\begin{tabular}{|c|c|c|}
\hline & \multicolumn{2}{|c|}{$\begin{array}{l}\text { Número total de mulheres } \\
\qquad=3440\end{array}$} \\
\hline & $\mathbf{N}$ & $\%$ \\
\hline \multicolumn{3}{|c|}{ Características individuais e condições sociodemográficas desfavoráveis } \\
\hline Gestantes com mais de 35 anos & 640 & 18,6 \\
\hline Gestantes com menos de 15 anos & 86 & 2,5 \\
\hline \multicolumn{3}{|l|}{ História reprodutiva anterior } \\
\hline Antecedente de DHEG/eclampsia & 59 & 1,7 \\
\hline Antecedente de prematuridade e baixo peso & 475 & 13,8 \\
\hline Antecedente de malformações fetais & 383 & 11,1 \\
\hline Antecedentes de neomorto e natimorto & 422 & 12,2 \\
\hline Abortamento habitual & 102 & 2,9 \\
\hline \multicolumn{3}{|c|}{ Condições clínicas preexistentes e intercorrências clínicas } \\
\hline Ginecopatias & 113 & 3,2 \\
\hline Hipertensão arterial crônica & 106 & 3,0 \\
\hline HIV & 112 & 3,2 \\
\hline Toxoplasmose & 115 & 3,3 \\
\hline Sífilis & 19 & 0,5 \\
\hline Rubéola & 24 & 0,7 \\
\hline Diabetes prévio & 7 & 0,2 \\
\hline Outras doenças infecciosas & 56 & 1,6 \\
\hline Outras doenças não infecciosas & 148 & 4,3 \\
\hline \multicolumn{3}{|l|}{ Doença obstétrica na gravidez atual } \\
\hline Gemelaridade & 161 & 4,6 \\
\hline Incompetência istmo-cervical & 91 & 2,6 \\
\hline Isoimunização $\mathrm{Rh}$ & 143 & 4,1 \\
\hline Malformações fetais & 812 & 23,6 \\
\hline Translucência nucal alterada & 119 & 3,4 \\
\hline Diabetes gestacional & 6 & 0,2 \\
\hline Pré-eclampsia e eclampsia & 20 & 0,6 \\
\hline Outras doenças obstétricas na gestação & 203 & 5,9 \\
\hline
\end{tabular}


receram em menos de $1 \%$ dos casos no grupo estudado.

Em relação às doenças obstétricas da gestação em curso, as malformações fetais se destacaram, sendo registradas em $23,6 \%$ dos casos, seguidas pela gemelaridade $(4,6 \%)$, isoimunização Rh $(4,1 \%)$, translucência nucal alterada $(3,4 \%)$ e incompetência istmo-cervical (2,6\%). Quando se analisa separadamente os tipos de malformações fetais mais prevalentes, encontramos $40 \%$ de defeitos relacionados ao sistema nervoso central e $18 \%$ renais (dados não apresentados em tabela).

$\mathrm{Na}$ análise bivariada (Tabela 3 ), a idade materna avançada e a gravidez em idade precoce (menos de 15 anos) foram fatores associados à renda familiar. Houve aumento do percentual de mulheres com idade materna acima de 35 anos na medida em que se observa aumento de rendimentos. Entre aquelas com menos de 15 anos, a relação com a renda foi inversa. Entre os demais fatores de risco reprodutivo, as ginecopatias e a translucência nucal alterada estiveram associadas às maiores faixas de renda; o HIV e as malformações fetais às faixas com menores rendimentos.

\section{Discussão}

O perfil econômico da clientela atendida no ambulatório de pré-natal do IFF/Fiocruz é de baixa renda, com cerca de dois terços das famílias (63\%) vivendo com no máximo três salários mínimos. No grupo estudado, 7,6\% das gestantes declararam renda familiar inferior a um salário mínimo, percentual equivalente aos $8 \%$ da população brasileira, que vivem em situação de extrema pobreza e que são alvo da política governamental de erradicação da miséria ${ }^{14}$.

Foi relevante o fato que $37,8 \%$ das mulheres atendidas no IFF serem oriundas de outros municípios/estados. Este achado pode denotar situações de vulnerabilidade relacionadas com dificuldade de acesso à assistência à saúde reprodutiva, uma vez que as gestantes de alto risco não conseguem ser incorporadas pela rede de saúde próxima de seu domicílio.

Os fatores de risco mais frequentes entre a clientela do pré-natal do IFF foram malformações fetais - também denominadas defeitos congênitos - na gravidez atual $(23,6 \%)$ e idade materna avançada $(18,6 \%)$, seguidos de histórico de problemas fetais em gravidez anterior, como prematuridade, baixo peso, neomorto, natimor-

Tabela 3. Perfil do risco reprodutivo segundo faixa de renda familiar de gestantes atendidas no ambulatório de pré-natal de alto risco do IFF/Fiocruz. 2006-2008.

\begin{tabular}{|c|c|c|c|c|c|c|c|}
\hline & \multicolumn{2}{|c|}{$\begin{array}{c}<1 \mathrm{SM} \\
\mathrm{N}=\mathbf{2 6 0}\end{array}$} & \multicolumn{2}{|c|}{$\begin{array}{c}1-3 \mathrm{SM} \\
\mathrm{N}=1890\end{array}$} & \multicolumn{2}{|c|}{$\begin{array}{c}4 \text { ou }+ \text { SM } \\
\mathrm{N}=935\end{array}$} & \multirow[t]{2}{*}{$\mathbf{p}$} \\
\hline & $\mathbf{N}$ & $\%$ & $\mathbf{N}$ & $\%$ & $\mathbf{N}$ & $\%$ & \\
\hline \multicolumn{8}{|c|}{ Características individuais e condições sociodemográficas desfavoráveis } \\
\hline Gestantes com mais de 35 anos & 32 & 12,3 & 307 & 16,3 & 258 & 27,6 & $<0,001$ \\
\hline Gestantes com menos de 15 anos & 16 & 6,2 & 50 & 2,6 & 6 & 0,6 & $<0,001$ \\
\hline \multicolumn{8}{|l|}{ História reprodutiva anterior } \\
\hline Antecedente de DHEG/eclampsia & 8 & 3,1 & 28 & 1,5 & 18 & 1,9 & 0,16 \\
\hline Antecedente de prematuro e baixo peso & 44 & 17,0 & 273 & 14,5 & 120 & 12,9 & 0,21 \\
\hline Antecedente de malformações fetais & 31 & 12,0 & 234 & 12,4 & 93 & 10,0 & 0,16 \\
\hline Antecedentes de neomorto e natimorto & 25 & 9,6 & 258 & 13,7 & 106 & 11,4 & 0,06 \\
\hline Abortamento habitual & 10 & 3,8 & 47 & 2,5 & 39 & 4,2 & 0,04 \\
\hline \multicolumn{8}{|c|}{ Condições clínicas preexistentes e intercorrências clínicas } \\
\hline Ginecopatias & 2 & 0,8 & 65 & 3,5 & 43 & 4,6 & 0,01 \\
\hline Hipertensão arterial crônica & 9 & 3,5 & 57 & 3,0 & 31 & 3,3 & 0,87 \\
\hline HIV & 17 & 6,6 & 55 & 2,9 & 21 & 2,3 & $<0,001$ \\
\hline Toxoplasmose & 13 & 5,0 & 64 & 3,4 & 30 & 3,2 & 0,3590 \\
\hline \multicolumn{8}{|l|}{ Doença obstétrica na gravidez atual } \\
\hline Gemelaridade & 9 & 3,5 & 91 & 4,8 & 46 & 4,9 & 0,59 \\
\hline Incompetência istmo-cervical & 7 & 2,7 & 53 & 2,8 & 26 & 2,8 & 0,99 \\
\hline Isoimunização Rh & 4 & 1,5 & 77 & 4,1 & 37 & 4,0 & 0,13 \\
\hline Malformações fetais & 69 & 25,6 & 453 & 23,9 & 181 & 19,3 & $<0,001$ \\
\hline Transluscência nucal alterada & 2 & 0,8 & 72 & 3,8 & 40 & 4,3 & 0,02 \\
\hline
\end{tabular}


to e malformações fetais, que ocorreram entre $11 \%$ e $14 \%$ dos casos. Este perfil de risco reprodutivo é condizente com a observação de Guerra et al. ${ }^{15}$ de que, embora não exista um sistema formalizado de referência para defeitos congênitos no Estado do Rio de Janeiro, o IFF é de fato a unidade de saúde para a qual, mais usualmente, são referidas as mulheres com gestações classificadas como de risco fetal e/ou genético.

Neste estudo, encontrou-se associação entre baixa renda familiar e maior ocorrência de malformações fetais. Condições socioeconômicas maternas desfavoráveis como baixa renda ${ }^{16,17}$, baixa escolaridade ${ }^{15,17,18}$ e carência nutricional ${ }^{18,19}$, têm sido associadas a maior prevalência de bebês com defeitos congênitos. No grupo estudado, foram predominantes as malformações fetais do sistema nervoso central (40\%), justamente aquelas que mais têm sido relacionadas com carência nutricional e exposições ambientais ${ }^{19}$.

Os defeitos congênitos têm adquirido grande relevância para a saúde pública, no Brasil e em outros países em desenvolvimento. Com maior controle de doenças infecciosas e nutricionais da infância, as malformações destacam-se entre as principais causas de mortalidade infantil ${ }^{16}$. No Brasil, os defeitos congênitos superaram as causas mal definidas, perinatais, infecciosas, nutricionais e respiratórias, tornando-se a segunda causa de mortalidade infantil ${ }^{19}$.

No estudo, encontrou-se associação entre renda familiar mais elevada e idade materna acima de 35 anos. Países desenvolvidos e em desenvolvimento têm demonstrado tendência de incremento de nascimentos entre mulheres com mais de 35 anos, nas últimas décadas ${ }^{20,21}$. No Brasil, as mulheres desse grupo etário representavam 7,6\% dos nascidos vivos, em 1994; esse percentual passou para $8,6 \%$, em 2000 e 10,1\% em $2009^{22}$. Maior escolaridade, participação no mercado de trabalho e outros espaços públicos, responsabilidades no sustento da família, complementando ou sendo principal provedora, e perspectiva de aperfeiçoamento profissional e formação de carreira tem sido fatores invocados como influentes no adiamento da maternidade ${ }^{23}$. O advento das novas tecnologias de reprodução assistida também tem concorrido para o fenômeno ${ }^{24}$.

O crescimento da reprodução na quarta e quinta décadas de vida traz novos desafios à saúde pública, exigindo desenho de novas linhas de cuidado para esse grupo específico. Estudos brasileiros demonstraram relação entre idade materna avançada e resultados perinatais desfavoráveis, inclusive aumento da morbimortalidade materna ${ }^{21,25,26}$. O risco obstétrico entre mulheres mais velhas está relacionado com maior frequência de doenças pré-existentes (hipertensão arterial, diabetes mellitus e outras) e de ocorrência de doenças específicas da gravidez (distocia, hipertensão induzida pela gestação, apresentações anômalas, oligodramnia, entre outros) $)^{21,27,28}$. A idade materna avançada também tem sido associada à anomalias cromossômicas ${ }^{15,18,29}$ e outras complicações fetais ${ }^{21,26}$.

Outro achado importante do estudo foi o maior percentual de gravidez antes dos 15 anos no grupo de renda familiar inferior a um salário mínimo. Molina et al. ${ }^{30}$ também encontraram forte associação entre essas variáveis, e ambas têm sido relacionadas à situações de vulnerabilidade individual, social e programática. Estudos realizados no Brasil e em outros países demonstraram associação entre gravidez adolescente e resultados perinatais desfavoráveis, como baixo peso ao nascer, prematuridade, mortalidade neonatal, pós-neonatal e no primeiro ano de vida ${ }^{31-}$ ${ }^{35}$, tendência mais proeminente entre as adolescentes de mais tenra idade ${ }^{35-40}$. Entre adolescentes menores de 15 anos, foram observadas ainda maiores taxas de mortalidade matern $a^{40}$.

Situações de vulnerabilidade social também têm sido identificadas entre mães adolescentes com menos de 15 anos. Pesquisa realizada por Santos ${ }^{41}$ com jovens desse grupo etário revelou desvinculação dos pais, desinformação sobre saúde sexual e reprodutiva e baixa escolaridade para a idade. Além disso, Teixeira e Taquette ${ }^{42}$ chamam a atenção para o fato de que meninas adolescentes são as principais vitimas de violência e exploração sexual, principalmente quando se encontram em situações de intensa vulnerabilidade, como aquelas que vivem em extrema pobreza. Essas adolescentes também estão expostas a outros tipos de violência e a maior exposição a doenças sexualmente transmissíveis ${ }^{42}$.

Pouca inserção em programas de saúde sexual e reprodutiva - necessidades contraceptivas não atendidas, início do pré-natal tardio, número reduzido de consultas pré-natal - delineiam um cenário de vulnerabilidade individual e programática das adolescentes mais jovens $s^{5,36,38,39,41,42}$.

A ocorrência do HIV entre as mulheres do pré-natal do IFF foi inversamente proporcional à renda familiar das gestantes, sendo esse fator de risco reprodutivo estatisticamente associado àquelas com menores rendimentos. A femininização e a pauperização do HIV/Aids tem sido um fenômeno observado desde meados dos anos de 
$1990^{43-46}$. Gênero e pobreza interagem na configuração de situações de menor acesso a recursos de saúde e educacionais, condições de negociação da prevenção menos favoráveis e maior exposição à violência ${ }^{46,47}$. A interseção entre pobreza, HIV e reprodução tem sido problemática, principalmente em face do desafio político, ético, legal e assistencial de garantia dos direitos reprodutivos de mulheres soropositivas ${ }^{41,48,49}$. Entre profissionais de saúde ainda há pouca compreensão desses direitos, e a assistência às necessidades em saúde reprodutiva das mulheres portadoras do HIV muitas vezes são permeadas por conflitos, especialmente quando existe o desejo de procriar ${ }^{41,49}$.

Outros achados com significância estatística foram as associações da translucência nucal (TN) alterada e das ginecopatias com o grupo de gestante de maior renda familiar, que como descrito anteriormente também está associado à idade materna avançada. A TN é um exame de rastreamento do primeiro trimestre da gestação que pode estar correlacionado às malformações estruturais $^{50,51}$. As ginecopatias contemplam uma série de alterações ginecológicas que afetam as mulheres como malformação uterina, miomatose, tumores anexiais e outros ${ }^{52}$. Na literatura revisada, não foram encontrados dados suficientes para interpretar de forma mais ampla essas associações. Contudo, em um contexto de desigualdades em saúde, renda mais elevada favorece $\mathrm{o}$ acesso a cuidados à saúde ginecológica $\mathrm{e}$ reprodutiva, incluindo início precoce e realização oportuna de exames pré-natal, repercutindo na qualidade da assistência à gestante ${ }^{53}$. Dessa maneira, os achados do presente estudo podem refletir a idade materna avançada e um maior acesso das mulheres com rendas superiores aos serviços de saúde, com maiores possibilidades de diagnósticos precoces.

$\mathrm{Na}$ pesquisa, o maior percentual de abortamento habitual ocorreu entre mulheres com renda familiar mais elevada, mas não há linearidade na ocorrência desse evento entre os três grupos de renda. Essa condição se define por história de perdas fetais sucessivas e espontâneas ${ }^{54}$, e sua etiologia é multifatorial envolvendo aspectos biológicos e ambientais ${ }^{55-57}$. Não foi encontrado na literatura, estudos que correlacionassem renda e abortamento habitual.

\section{Conclusão}

O estudo do perfil do risco reprodutivo e de sua associação com condições socioeconômicas, com base no banco de dados de admissão no ambulatório de pré-natal do IFF, apresenta limitações. O formulário contem somente duas variáveis socioeconômicas, renda e escolaridade, porém a última não havia sido preenchida em cerca de metade dos casos, comprometendo a análise. Não havendo registro do número de membros da família que residem no mesmo domicilio, não foi possível calcular a renda familiar per capita, o que teria permitido maior comparabilidade com análises dos indicadores sociais do Instituto Brasileiro de Geografia e Estatística (IBGE). Além disso, apesar da importância das associações encontradas, não é possível generalizar o perfil de risco reprodutivo tendo em vista peculiaridades do serviço estudado que, por sua tradição na área de pediatria, neonatologia e genética médica, se volta principalmente para a assistência à gravidez de risco fetal e neonatal.

A reflexão sobre como discriminações étnicoraciais podem influenciar a distribuição dos riscos reprodutivos foi impossibilitada pela ausência de registro da cor de pele autodeclarada no formulário do banco de dados. Outras investigações devem se debruçar sobre essa questão uma vez que estudos com populações de mulheres negras mostram que elas apresentam piores indicadores de saúde reprodutiva ${ }^{58}$ e maiores desigualdades no acesso e utilização de serviços de saúde ${ }^{59}$.

As variáveis do banco de dados tampouco permitem uma investigação ampla de como desigualdades de gênero podem estar associadas a situações de vulnerabilidade individual, social e programática no que concerne à saúde reprodutiva. Gestores e profissionais de saúde ainda têm grandes dificuldades de incorporar questões de gênero em suas práticas, fato que se reflete nos processos de trabalho nos serviços de saúde. Por exemplo, nas consultas pouco se abordam questões relacionadas ao contexto de vida das mulheres e pouca atenção se dá a situações como dupla jornada de trabalho, violência doméstica, assédio sexual ou moral no trabalho, dependência econômica, chefia de lar monoparental e outras que podem condicionar as práticas de cuidado, o acesso e a utilização de serviços e, enfim, a satisfação das necessidades em saúde. Apesar das evidências, o registro de informação referente ao contexto social ainda é pouco valorizado por gestores e profissionais de saúde. Transformar essas questões em variáveis que podem ser estudadas com metodologia quantitativa é um desafio ainda maior.

Apesar das limitações do estudo, consideramos que seu resultado é relevante ao chamar a atenção para a necessidade de maior conheci- 
mento do perfil do risco reprodutivo das mulheres brasileiras e sua distribuição segundo características sociodemográficas e econômicas específicas. A baixa renda se associou nitidamente com alguns riscos reprodutivos, levantando questões sobre como vulnerabilidades sociais e programáticas podem operar na produção das situações de risco à saúde reprodutiva e quais desafios são colocados aos serviços de saúde em face das mesmas.

Outros indícios de vulnerabilidades em saúde sexual e reprodutiva que despontam do estudo precisam ser mais investigados. Neste de universo de mulheres com gravidezes classificadas como de alto risco, há um grupo expressivo com histórico de múltiplas gestações e/ou de abortamento, levando à indagação sobre a satisfação das necessidades de planejamento reprodutivo de cuidados com saúde reprodutiva dessas mulheres. Ao estudar mulheres aloimunizadas matriculadas neste mesmo serviço de pré-natal, Assumpção ${ }^{60}$ encontrou elevado número de multigestas e grandes multíparas, cujas histórias revelaram a falta de assistência à saúde reprodutiva e planejamento reprodutivo nos intervalos entre as gravidezes.
A elevada frequência de gestantes que vivem com HIV observada merece também ser investigada em pesquisas futuras, pois ela tanto pode refletir situações de vulnerabilidade individual e programática (falha na assistência à contracepção e prevenção das doenças sexualmente transmissíveis), como pode significar avanços na garantia dos seus direitos reprodutivos.

Enfim, cuidados com a saúde nos períodos fora da gravidez e durante o pré-natal desempenham papel fundamental no controle dos fatores de risco reprodutivo e na redução da morbimortalidade materna e perinatal. Contudo, se os riscos reprodutivos são influenciados por questões que ultrapassam o âmbito biomédico e dizem respeito às vulnerabilidades sociais, a instituição de saúde deve ultrapassar os limites das práticas assistenciais focadas exclusivamente em doenças ou riscos biológicos e desenvolver abordagens que possam enfrentar as desigualdades sociais em saúde. Uma vez que o adoecimento carrega junto consigo questões de classe social, gênero, raça/etnia, entre outras, é fundamental que se incorporem no cotidiano dos profissionais de saúde discussões sobre direitos fundamentais, cidadania e equidade.

\section{Colaboradores}

RB Xavier, CB Jannotti e KS Silva participaram da concepção do projeto, da coleta e da análise dos dados e da redação do artigo. AC Martins participou da etapa final de redação do artigo. Todos os autores revisaram a versão final do artigo e concordaram com sua submissão. 


\section{Referências}

1. Brasil. Ministério da Saúde (MS). Assistência prénatal, manual técnico. Brasília: Secretaria de Políticas de Saúde, MS; 2000.

2. Brasil. Ministério da Saúde (MS). Parto, aborto e puerpério, assistência humanizada à mulher. Brasília: Secretaria de Políticas de Saúde, MS; 2001.

3. Brasil. Ministério da Saúde (MS). Política Nacional de Atenção Integral à Saúde da mulher: princípios e diretrizes. Brasília: Secretaria de Atenção à Saúde, MS; 2004.

4. Brasil. Ministério da Saúde (MS). Manual técnico de pré-natal e puerpério, atenção qualificada e humanizada. Brasília: Secretaria de Políticas de Saúde, MS; 2005.

5. Brasil. Ministério da Saúde (MS). Manual gestação de alto risco. Brasília: Secretaria de Políticas de Saúde, MS; 2010.

6. Escalante JJC, Viola RC, Alves SV. Mortalidade materna no Brasil. In: Brasil. Ministério da Saúde (MS). Saúde Brasil 2009: uma análise da situação de saúde e da agenda nacional e internacional de prioridades em saúde. Brasília: Secretaria de Vigilância em Saúde; 2010. p. 201-221.

7. Escalante JJC, Morais Neto OL. A redução da mortalidade na infância e infantil no Brasil e nas unidades da federação. In: Brasil. Ministério da Saúde (MS). Saúde Brasil 2009: uma análise da situação de saúde e da agenda nacional e internacional de prioridades em saúde. Brasília: Secretaria de Vigilância em Saúde; 2010. p. 177-199.

8. Giffin K. Pobreza, desigualdade e eqüidade em saúde: considerações a partir de uma perspectiva de gênero transversal. Cad Saude Publica 2002; 18(Supl.):103-112.

9. Chor D, Lima CRA. Aspectos epidemiológicos das desigualdades raciais em saúde no Brasil. Cad Saude Publica 2005; 21(5):1586-1594.

10. Soares VMN, Souza KV, Freygang TC, CV, Saito MR. Mortalidade materna por pré-eclâmpsia/ eclâmpsia em um estado do Sul do Brasil. Rev Bras Ginecol Obstet 2009; 31(11):566-573.

11. Morse ML, Fonseca SC, Barbosa MD, Calil MB, Eyer FPC. Mortalidade materna no Brasil: o que mostra a produção científica nos últimos 30 anos? Cad Saude Publica 2011; 27(4):623-638.

12. Brasil. Ministério da Saúde (MS). Manual dos comitês de mortalidade materna. Brasília: Secretaria de Atenção à Saúde, MS; 2007.

13. Ayres JR, França Júnior I, Calazans GJ, Saletti Filho HC. Vulnerabilidade e prevenção em tempos de Aids. In: Barbosa RM, Parker R, organizadores. Sexualidades pelo avesso: direitos, identidades e poder. Rio de Janeiro, São Paulo: IMS, UERJ; 1999. p. 49-72.

14. Brasil. Ministério do Desenvolvimento Social (MDS) Plano Brasil sem Miséria. [site da Internet][acessado em 2011 out 7]. Disponível em: http://www.brasil semmiseria.gov.br/conheca-o-plano/

15. Guerra FAR, Llerena Júnior JC, Gama SGN, Cunha CB, Theme Filha MM. Defeitos congênitos no município do Rio de Janeiro, Brasil: uma avaliação através do SINASC (2000-2004). Cad Saude Publica 2008; 24(1):140-149.
16. Cornel CM. Wealth and health in relation to birth defects mortality. J Epidemiol Community Health 2000; 54(9):644

17. Yang J, Carmichael SL, Canfield M, Song J, Shaw GM. Socioeconomic status in relation to selected birth defects in a large multicentered US case-control study. Am J Epidemiol 2008; 167(2):145-154.

18. Costa CMS. Perfil das malformações congênitas em uma amostra de nascimentos no Município do Rio de Janeiro, 1999-2001 [dissertação]. Rio de Janeiro: Escola Nacional de Saúde Pública, Fundação Oswaldo Cruz; 2005.

19. Horovitz DDG, Llerena Júnior. JC, Mattos RA. Atenção aos defeitos congênitos no Brasil: panorama atual. Cad Saude Publica 2005; 21(4):1055-1064.

20. Cleary-Goldman J, Malone FD, Vidaver J, Ball RH, Nyberg DA, Comstock CH, Saade GR, Eddleman KA, Klugman S, Dugoff L, Timor-Tritsch IE, Craigo SD, Carr SR, Wolfe HM, Bianchi DW, D'Alton M, Faster C. Impact of maternal age on obstetric outcome. Obst Gynecol 2005; 105(5 Pt 1):983-990.

21. Santos GHN, Martins MG, Sousa MS, Batalha SJC. Impacto da idade materna sobre os resultados perinatais e via de parto. Rev Bras Ginecol Obstet 2009; 31(7):326-334.

22. Brasil. Ministério da Saúde (MS). Departamento de Informática do SUS. Informações de saúde, demográficas e socioeconômicas. [site da Internet][acessado 2011 set 17]. Disponível em: http://tabnet.datasus. gov.br/cgi/tabcgi.exe?sinasc/cnv/nvuf.def.

23. Parada CMGL, Tonete VLP. Experiência da gravidez após os 35 anos de mulheres com baixa renda. Esc Anna Nery 2009; 13(2):385-392.

24. Friese C, Becker G, Nachtigall RD. Older motherhood and the changing life course in the era of assisted reproductive technologies. J Aging Stud 2008; 22(1):65-73

25. Azevedo GD, Freitas Júnior RAO, Freitas AKMSO, Araújo ACPF, Soares EMM, Maranhão TMO. Effect of maternal age on perinatal outcomes. Rev Bras Ginecol Obstet 2002; 24(3):181-185.

26. Andrade PC, Linhares JJ, Martinelli S, Antonini M, Lippi UG, Baracat FF. Resultados perinatais em grávidas com mais de 35 anos: estudo controlado. Rev Bras Ginecol Obstet 2004; 26(9):697-701.

27. Costa HLFF, Costa CFF, Costa LOBF. Idade materna como fator de risco para a hipertensão induzida pela gravidez: análise multivariada. Rev Bras Ginecol Obstet 2003; 25(9):631-635.

28. Cecatti JG, Faúndes A, Surita FGC, Aquino MMA O Impacto da idade materna avançada sobre os resultados da gravidez. Rev Bras Ginecol Obstet 1998; 20(7):389-394.

29. Gusmão FAF, Tavares EJM, Moreira LMA. Idade materna e síndrome de down no nordeste do Brasil. Cad Saude Publica 2003; 19(4):973-978.

30. Molina CR, Molina GT, González AE. Madres niñasadolescentes de 14 años y menos: Un grave problema de salud pública no resuelto en Chile. Rev Med Chile 2007; 135(1):79-86 
31. Oliveira EF, Gama SGN, Silva CMFP. Gravidez na adolescência e outros fatores de risco para mortalidade fetal e infantil no Município do Rio de Janeiro, Brasil. Cad Saude Publica 2010; 26(3):567-578.

32. Chen XK, Wen SW, Fleming N, Yang Q, Walker MC. Increased risks of neonatal and postneonatal mortality associated with teenage pregnancy had different explanations. J Clin Epidemiol 2008; 61(7): 688-694.

33. Chalem E, Mitsuhiro SS, Ferri CP, Barros MCM, Guinsburg R, Laranjeira R. Gravidez na adolescência: perfil sócio-demográfico e comportamental de uma população da periferia de São Paulo, Brasil. Cad Saude Publica 2007; 23(1):177-186.

34. Gama SGN, Szwarcwald CL, Leal MC, Theme Filha MM. Gravidez na adolescência como fator de risco para baixo peso ao nascer no Município do Rio de Janeiro, 1996 a 1998. Rev Saude Publica 2001; 35(1): 74-80.

35. Fraser AM, John EMSPH, Brockert, MPH, Ward $\mathrm{RH}$. Association of young maternal age with adverse reproductive outcomes. N Engl J Med 1995; 332(17):1113-1118.

36. Goldenberg P, Figueiredo MCT, Silva R. Gravidez na adolescência, pré-natal e resultados perinatais em Montes Claros, Minas Gerais, Brasil. Cad Saude Publica 2005; 21(4):1077-1086.

37. Magalhães MLC, Furtado FM, Nogueira MB, Carvalho FHC, Almeida FML, Mattar R, Camano L. Gestação na adolescência precoce e tardia: há diferença nos riscos obstétricos? Rev Bras Ginecol Obstet 2006; 28(8):446-452.

38. Simões VF, Silva AAM, Bettiol H, Lamy-Filho F, Tonial SR, Mochel EG. Características da gravidez na adolescência em São Luís, Maranhão. Rev Saude Publica 2003; 37(5):559-565.

39. Phipps MG, Sowers MF. Defining Early Adolescent Childbearing. Am J Public Health 2002; 92(1):125128.

40. Donoso SE, Becker VJ, Villarroel PL. Natalidad y riesgo reproductivo en adolescentes de Chile, 19901999. Rev Panam Salud Publica 2003; 14(1):3-8

41. Santos ALD. Historia de Jovens Que Vivenciaram a Maternidade na Adolescência Menor [tese]. São Paulo: Faculdade de Saúde Pública, Universidade de São Paulo; 2006.

42. Teixeira SAM, Taquette SR. Violência e atividade sexual desprotegida em adolescentes menores de 15 anos. Rev Assoc Med Bras 2010; 56(4):440-446.

43. Parker R, Camargo Júnior KR. Pobreza e HIV/AIDS: aspectos antropológicos e sociológicos Cad Saude Publica 2000; 16(Supl. 1):89-102.

44. Bastos FI, Szwarcwald CL. Aids e pauperização: principais conceitos e evidencias empíricas. Cad Saude Publica 2000; 16(Supl. 1):65-76.

45. Barbosa LM, Sawyer DO. AIDS: a vulnerabilidade social e a evolução da epidemia nos municípios das regiões Nordeste e Sudeste do Brasil. Rev Bras Estud Popul 2003; 20:241-257.

46. Brasil. Ministério da Saúde (MS). Plano integrado de enfrentamento à feminização da epidemia de AIDS e outras DST. Brasília: Secretaria de Vigilância em Saúde, MS; 2007.
47. Miranda-Ribeiro P, Simão AB, Caetano AJ, Lacerda MA, Torres EA. Perfis de Vulnerabilidade Feminina ao HIV/AIDS em Belo Horizonte e Recife: comparando brancas e negras. Saude soc 2010; 19(Supl. 2):21-35.

48. Sant'anna ACC, Seidl EMF. Efeitos da condição sorológica sobre as escolhas reprodutivas de mulheres HIV positivas. Psicol Reflex Crit 2009; 22(2): 244-251.

49. Paiva V, Lima TN, Santos N, Ventura-Filipe E, Segurado A. Sem direito de amar? A vontade de ter filhos entre homens (e mulheres) vivendo com o HIV. Psicol USP 2002; 13(2):105-133.

50. Camano L, Moron AF, Nardozza LM, Machado, PDBS, Chinen PA. Avaliação do desfecho dos conceptos com risco aumentado de ocorrência de anomalias cromossômicas calculado pela medida da translucência nucal. Rev Bras Ginecol Obstet 2005; 27(3):155-160.

51. Saldanha FAT, Brizot ML, Lopes LM, Liao AW, Zugaib M. Anomalias e prognóstico fetal associados à translucência nucal aumentada e cariótipo anormal. Rev Assoc Med Bras 2009; 55(1):54-59.

52. Freitas FM, Rodini GP, Santos C. Ginecopatias. In: Montenegro CAB, Rezende Filho J, organizadores. Rezende Obstetrícia. Rio de Janeiro: Guanabara koogan; 2010. p. 760-768.

53. Gonçalves CV, Cesar JA, Mendoza-Sassi RA. Qualidade e eqüidade na assistência à gestante: um estudo de base populacional no sul do Brasil. Cad Saude Publica 2009; 25(11):2507-2516.

54. Stirrat GM. Recurrent miscarriage. I. Definition and epidemiology. Lancet 1990; 336(8716):673-675.

55. Bricker L, Farquharson RG. Types of pregnancy loss inrecurrent miscarriage: implications for research and clinical practice. Hum Reprod 2002; 17(5):1345-1350.

56. Christiansen OB, Nielsen HS, Kolte A, Pedersen AT. Researchmethodology and epidemiology of relevance in recurrentpregnancy loss. Semin Reprod Med 2006; 24(1):5-16.

57. Montenegro CAB, Rezende Filho J. Abortamento. In: Montenegro CAB, Rezende Filho J, organizadores. Rezende Obstetrícia. Rio de Janeiro: Guanabara Koogan; 2010. p. 354-368.

58. Martins AL. Mortalidade materna de mulheres negras no Brasil. Cad Saude Publica 2006; 22(11):24732479.

59. Leal MC, Gama SGN, Cunha CB. Desigualdades raciais, sociodemográficas e na assistência ao prénatal e ao parto, 1999-2001. Rev Saude Publica 2005; 39(1):100-107.

60. Assumpção AMB. Relatos de gestantes aloimunizadas: Uma reflexão sobre contextos de vida e riscos a saúde reprodutiva [dissertação]. Rio de Janeiro: Instituto Fernandes Figueira, Fundação Oswaldo Cruz; 2006.

Artigo apresentado em 08/11/2011

Aprovado em 28/02/2012

Versão final apresentada em 31/03/2012 
